\title{
James Steinhoff
}

jsteinh@uwo.ca

\section{Towards a Political Economy of Synthetic Data: A Data-intensive Capitalism that is not a Surveillance Capitalism? [PREPRINT. Forthcoming in New Media \& Society]}

Surveillance is the unspoken byword of contemporary capitalism. New business models driven by data-intensive technologies like machine learning rely on the collection of large quantities of data, much of which is obtained by surveilling users of social media, mobile devices and applications. The capitalist drive to accumulation is increasingly funneled through this dataintensive infrastructure, making surveillance a prerequisite for profit-generation. The search for new markets takes the form of continual incursions of surveillance into new spheres of life, driving visions of a future characterized by increasingly fine-grained and omnipresent monitoring. A social backlash, fueled in part by such visions, has contributed to the establishment of regulations on the collection and usage of data, and thus on surveillance, such as the European Union's General Data Protection Regulation, implemented in 2018. Against such developments, data-intensive firms are striving to maintain the ability to collect data through surveillance via techniques ranging from record-setting lobbying spending (Chung, 2021) to encouraging a digital resignation to surveillance in users (Draper and Turow, 2019). This paper examines another, technical, way in which data-intensive capital is responding to the curtailment of surveillance: synthetic data.

Synthetic data is data which is not collected via surveillance; rather it is 'produced artificially' (Nikolenko, 2021: v). It is data 'that computer simulations or algorithms generate as an alternative to real-world data' (Andrews, 2021). This paper introduces and critically assesses this technology from a political economy perspective, drawing on analysis of documents from machine learning, data science and computer science research and the artificial intelligence industry. It is easy to imagine why data-intensive capital might find synthetic data appealing at a moment of heightened scrutiny over its surveillance practices. However, I argue that the appeal of synthetic data for data-intensive capital goes beyond providing an alternative to surveillance. It also provides a novel technical means for continuing a historical tendency within capitalism towards the autonomization of the circuit of capital. The autonomization of capital is most often discussed in terms of the automation of production and circulation processes, as well as the speculative movements of value in the finance sector. But by shifting the production of data from a process contingent on the surveillance of human subjects to one of computational generation, synthetic data contributes to the automation of the production of the conditions for production and circulation in data-intensive capitalism. This, I argue, presents ontological, epistemological and political implications for data-intensive technologies, data-intensive capital and struggles against it.

\section{Context: Data-intensive Capital and Surveillance}


Capitalism is the mode of production founded on the process of transforming a quantity of value into more value via the production and exchange of commodities. Karl Marx schematized this process as: M-C-M' (Marx, 1990: 251). More value must be generated than was invested; capital must be 'valorized' (Marx, 1990: 252). Capital is this process endlessly repeated; it is an 'end in itself' (Marx, 1990: 253). Hence the restlessness of capitalism. Valorization can never be finally completed. New markets must always be opened and new commodities produced. But wherein does value arise? According to Marx, and contrary to contemporary mainstream economics, not from the sale of commodities for more than their production costs. Rather, the value of capital arises from exploitation, which in Marx's technical sense refers to the difference between the wage paid to a worker and the greater value that worker's capacity to labour can produce (Marx, 1990: 418-421). Capitalists aim to maximize this difference, which Marx calls "surplus-value" (Marx, 1990: 293). The unequal exchange of capacity to labour for a wage defines the immanent antagonism of capitalism, between labour and capital.

Yet value is an abstract quantity. It can only exist if there are commodities which may be valued. Thus the process of valorization necessitates a labour process, in which the concrete actions of humans produce goods or services. In the labour process, the labour/capital antagonism finds its primal expression. Since capital as such consists of numerous individual capitals engaged in competition, those individual capitals are driven to optimize their labour processes so as to increase the productivity of labour and the amount of surplus-value captured relative to their peers (Marx, 1990: 433). There are many ways to achieve increased productivity of labour going back to the division of labour. But Marx singled out the introduction of machinery as a qualitative break, because machines can be continually revolutionized, presenting ever new possibilities for increased productivity versus competitors (Marx, 1990: 1035). Capital's imperative to valorization, driven by competition, thus compels the transformation of labour-intensive production processes into 'process[es] of the technological application of scientific knowledge' (Marx, 1990: 775). In contemporary terms: processes of automation.

As Ramtin (1991) argues, once it is possible, automation becomes an 'objective necessity imposed by the very functioning of the capitalist mode of production itself, in accordance with and as a result of the law of value' (101). This does not mean that automation proceeds in a linear fashion, progressively eliminating humans across the economy. Rather, the automation of one task may necessitate new types of labour in adjacent tasks (Gray and Suri, 2019: 206; Delfanti and Frey, 2021). But wherever such new pockets of labour appear within data-intensive capital, there also appear efforts to replace them with machines, as the contemporary AI industry demonstrates (Steinhoff, 2021: 193-200). Over time, the constant component of capital (including machines) increases while its human component, labour, or variable capital, decreases. Marx (1990) referred to this as the increasing 'organic composition' of capital (762). This concept plays a central role in Marx's schema, explaining several of capital's distinctive macro-level tendencies (Ross, 2015). However it also manifests on the smaller scale of the labour process. 
In the labour process, machines are used to increase the speed and volume of production, but also to wrest control over the labour process from labour and transfer it to capital. Braverman (1998) describes this as the 'separation of conception from execution' (79). Its larval form is the division of labour, which develops into the 'progressive elimination of the control functions of the worker ... and their transfer to a device which is controlled ... by management from outside the direct process' (Braverman, 1998: 146). Case studies of this separation abound in industries from machine tools (Noble, 1986) to education software (Mirrlees and Alvi, 2019). Machines allow capital to administer control over labour at an infrastructural level, fixing parameters of possible action and precluding alternative courses. The final stage of control is wholesale automation, or the 'dissociation of living labour ... from the production process' (Ramtin, 1991: 58). While such a maximally organic composition of capital is rarely obtained for most labour processes, it remains a motivating principle by structural necessity: the 'development of the means of labour into machinery is not an accidental moment of capital, but is rather the historical reshaping of the traditional, inherited means of labour into a form adequate to capital' (Marx, 1993: 694, emphasis added). A rising organic composition renders the substrate of the labour process qualitatively more adequate to the valorization process of capital, insofar as it minimizes the limitations, contingencies and resistances potentially posed by labour's human subjectivity.

2007 was a significant year in terms of the organic composition of capital. That year, Google acquired the targeted-advertising company DoubleClick, "ushering in a massive agglomeration of data between Google's search information and DoubleClick's user-tracked marketing data", forming an "Internet-wide surveillance network" and setting off a chain of similar data-intensive acquisitions (Cheney-Lippold, 2017: 20). Since then, many sectors of capital have incorporated data-intensive technologies into their operations. Indeed, contemporary capitalism is often defined by its relation to data. It may be in terms of a 'platform capitalism' centered on 'extracting and using a particular kind of raw material: data' (Srnicek, 2017). It might be in terms of a 'data capitalism' premised on 'the sale of individual behavioral profiles tied to user data' (West, 2019: 23). Or it may be in terms of a 'surveillance capitalism' since data collection depends on the 'unilateral surveillance ... of human behavior' (Zuboff, 2016: 1). The notion is one of a new mode of capitalist production in which digital data, harvested via surveillance, is of central importance to valorization. ${ }^{1}$ Data-intensive technologies, it would seem, are quite adequate to capital.

While few employ Marxist terminology, critical scholars of data and artificial intelligence (AI) have tracked the increasingly data-intensive composition of capital. Such work has shown how data-intensive technologies are anything but neutral, immaterial and inhuman. Rather, such scholars have argued that data-intensive technologies are implicated deeply in existing social relations, depend for their existence on a rich array of material factors and cannot be considered in abstraction from the humans who design, deploy and use them. More specifically, critical scholars have demonstrated how data-intensive technologies reinforce existing prevalent forms of discrimination (Noble, 2018; Katz, 2020) while undermining and complicating democratic processes (Sudmann, 2019), reconfiguring epistemologies (Kitchin, 2014; Lepage-Richer, 2021) 
and bolstering the pathologies of the capitalist mode of production, from ecological devastation to colonial predation of labour and resources (Chun, 2018; Dyer-Witheford et al., 2019; Verdegem, 2021; Crawford, 2021; Halpern, 2021). Others have pointed out how a selfreinforcing dynamic has developed between data and capital.

Once data-intensive technologies are built-in to business models, data becomes subject to the same endless self-justification as valorization. Data-intensive capital must 'constantly collect and circulate data by producing commodities that create more data and building infrastructure to manage data. The stream of data must keep flowing and growing' (Sadowski, 2019: 4). Andrejevic (2020) notes a 'cascading logic of automation ... [in which] automated data collection leads to automated data processing, which, in turn, leads to automated response' (9). While social media presents a wealth of data, capital cannot stop there, so surveillance extends 'deeper into areas of human life that were once off-limits or too expensive to reach' (Crawford, 2021: 119). The notion is that, barring resistance, omnipresent, or as Andrejevic (2020) puts it, 'frameless', surveillance awaits. Capital's data hunger will require an increasingly rigorous tracking of the human subjects from which data is generated. Thus, while data-intensive capital may achieve new levels of organic composition, a human element remains at its core.

Early critical research on data pointed out that data does not simply exist fully-formed in the wild; it must be created. For Manovich (2001), data 'does not just exist - it has to be generated. Data creators have to collect data and organize it, or create it from scratch' (224). For Bowker (2005), '[r]aw data is both an oxymoron and a bad idea' (184). Rather, data must be 'cooked' or prepared. Subsequent work would also reject the notion of raw data (Gitelman, 2013), but would drop Manovich's suggestive reference to creating data from scratch. Data is now recognized as the product of sensors collecting the traces of human actions; a copy of the world, not something that can be created from scratch. For Sadowski (2019) data is a 'recorded abstraction of the world' (2) much of which is 'about people' (6). For, Gregory (2014) data 'is made of people' or more precisely, the 'very rhythms, circulations, palpitations, and mutations of our bodies'. For Lemov (2016) big data 'is people' because 'data is not only generated about individuals but also made out of individuals' (emphasis original). Data possesses an 'inherent humanness' since it is premised on the 'almost literal mining of subjectivity' (Lemov, 2016, emphasis original) or the extraction of 'human experience as free raw material' (Zuboff, 2019: 5). This premise has been carried over into critical AI studies, where it is held that the 'data used today in ML, and especially DL, is all too human' (Roberge and Castelle, 2021: 10) and that 'AI should be owned by the people, because it is overwhelmingly "fed" by the people' (Dippel, 2021: 40).

In sum, critical views on data insist that data's provenance is human subjectivity. ${ }^{2}$ Surveillance is thus not an accidental component of data-intensive capital, but its condition of possibility. While I do not dispute this logic, my goal is to explore an alternative means by which data-intensive capital might satisfy its hunger for data. What if capital could obtain data from a means other than the surveillance of people? This is the possibility suggested by synthetic data. 


\section{Synthetic Data}

Synthesis describes an act of combination; bringing together elements to generate a new whole; a 'process that, by human agency, emulates certain properties of a naturally occurring material' (Raghunathan, 2021: 131). While natural rubber, made from the latex of the hevea brasiliensis tree, remains a component of automobile tires, it has been superseded by synthetic rubber, produced by chemical synthesis of petroleum products. Synthetic rubber emulates natural latex and attenuates the connection between tires and hevea brasiliensis. Synthetic data is described as 'data that is artificially created rather than being generated by actual events' (Dilemgani, 2021). It is data which is not a trace, copy or recording, but the product of a computational process. Synthetic data thus purports to attenuate the connection between data and people by synthesizing data. While all data are to some degree synthetic since they are not found fully-formed, the contemporary phenomenon of synthetic data is differentiated by its disconnection from the socalled real world. As I will show, this obtains as a matter of degrees. Different approaches to synthetic data are more or less disconnected from human subjects and the real world-and none are completely divorced.

Before delving into the technical details, it is worth considering why there is interest in making even more data, living as we are amidst an unprecedented abundance or surfeit of data (Mosco, 2015; Andrejevic, 2013). In short, from the perspective of machine learning and computer science research, there is no surfeit, but rather a dire lack of data. Computer scientists complain of a 'data shortage' (Yang, 2019) and 'data scarcity' (Bansal, Sharma and Kathuria, 2020) and assert that 'many problems of modern AI come down to insufficient data' (Nikolenko, 2021: 12). Unsurprisingly, since AI is now integrated into many business processes, industry commentators share this perspective. A writer for MIT Sloan Management Review discusses a 'real challenge many companies face: the lack of enough data to train a model' (Lucini, 2021). According to one recent study, $96 \%$ of companies aiming to implement machine learning applications report running into problems pertaining to data quantity and quality (Dimensional Research, 2019).

There are three frequently cited causes of the data shortage in both the technical and business literature. The first derives from the fact that data for supervised learning, the most popular approach to machine learning, must be hand-labeled (Nikolenko, 2021: 12). This is labour-intensive and makes creating labeled datasets difficult and buying them costly. One industry blogger puts the cost of a quality dataset between $\$ 10,500$ to $\$ 85,000$ USD (Incze, 2019). Further, hand-labeling is unreliable. Recent research has shown that several datasets widely used as benchmarks contain substantial amounts of errors (Wiggers, 2021a). The second cited cause of the data shortage is that for some applications sufficient data simply do not exist. One may think of data in relation to digital platforms where millions of interactions can be captured each day, but for many potential applications of machine learning (farming is often mentioned) there is very little data (Yang, 2019; DeepLearningAI, 2021). A related concern here is bias. While bias in data is a deep problem with diverse social factors (Ntoutsi et al., 2020), 
from a technical perspective it refers to a dataset inadequately representing a particular feature. Synthetic data presents the possibility of creating new data to fix datasets biased in this sense (Watson, 2020). The third cited cause of the data shortage pertains to data accessibility. This refers to data which exists, but is rendered inaccessible by social, rather than technical, factors including privacy concerns, proprietary ownership and regulation (El Emam, 2021). Medical applications are frequently mentioned in this regard. To summarize, from the perspectives of computer science and capital, there is no data surfeit, but rather a data shortage. Data is rare, expensive and time-consuming to label, and access to the data that exists is often difficult, impossible or ethically unsound. This is the context in which synthetic data has been commercialized.

While synthetic data is far from superseding conventional data and far from eliminating completely the human component from the production of data, it is also far from merely theoretical. At the time of writing in early 2022, there are over 15 startup companies with synthetic data commodities on the market, including Synthesis AI, DataGen, Anyverse, Trūata and Mostly.AI. Large tech companies are also involved. Microsoft (nd) plans to open-source its Synthetic Data Generator and in 2021, Facebook spent an undisclosed sum to acquire the startup AI.Reverie, which creates simulated environments (Wiggers, 2021b). ${ }^{3}$ Even large non-AI companies like J.P. Morgan, John Deere and American Express are producing and using synthetic data internally.

Still, one might reasonably ask: does synthetic data actually work? Experimental results suggest that it does. Using a generative model approach (detailed below) researchers created synthetic datasets from five publicly available datasets and challenged data scientists to develop predictive models from them. They compared the results of the data scientists working with the synthetic data to that of data scientists working with the original data and found "no significant difference" suggesting that "synthetic data can successfully replace original data for data science" (Patki, Wedge and Veeramachaneni, 2016: 399). More recent studies are also supportive. Rankin et al. (2020) hold that most (92\%) of models trained on synthetic data have only slightly lower accuracy than those trained on real data while Foraker at al. (2020) find that analyses on both synthetic and real data produced sufficiently statistically similar conclusions.

With context now established, it is time to turn to synthetic data itself. I will discuss three approaches to producing it: data augmentation, generative models and simulated environments. I find it useful to imagine these on a continuum, in the same order, going from least to most synthetic, defined heuristically as how removed the data they produce are from the surveillance of human subjects. These approaches are often combined. For instance, you might generate synthetic data by capturing images of 3D faces in a simulated environment. You might produce these faces, in turn, with a generative model, and you might expand your set of captured images via data augmentation.

\section{Data Augmentation}


Data augmentation is the automated application of minor modifications to a dataset in order to enlarge or diversify it. This is most readily explicated in terms of image data. Here augmentation can be as simple as rotating, cropping or blurring images. Even if you merely rotate each existing photo once, you can double the size of your dataset. The potential gains are, however, much larger. Krizhevsky, Sutskever and Hinton (2012) show that 2048 new images can be generated from a single input image via data augmentation (5). Further, since labels are preserved throughout augmentation, a labeled dataset can be expanded for no additional labeling cost. The simplicity and effectiveness of data augmentation have made it a widespread practice. Today, most state-of-the-art image recognition models are trained with augmented data.

Data augmentation may not sound very synthetic, as it merely extrapolates from existing data. However, it presents an example of how data can be procured, if not from scratch, from the manipulation of existing data. And when machine learning is applied to data augmentation it further blurs the line between data augmentation and generation (Nikolenko, 2021: 90). So-called 'smart' augmentation involves having a network which does not simply apply preset augmentations but learns an optimal way to augment data in the course of training in accord with reducing another network's error (Lemley, Bazrafkan and Corcoran, 2017).

\section{Generative Models}

Generative models generate a novel output, rather than classify a given input as this or that category (i.e. object recognition). There are many types of generative models, but the kind most used for synthetic data generation are generative adversarial networks (Goodfellow et al., 2014). The technical details are beyond the ambitions of this paper, but the basic idea is to pit two networks against one another, with one trying to get the other to 'misclassify data ... that are only marginally different from those that they adequately classify' (Lepage-Richer, 2021: 215). A model can be trained over the course of such adversarial exchanges that can extract and reproduce the statistical properties (and labels) of interest of a real dataset in a new synthetic dataset. Sensitive aspects may be eliminated, such that the new dataset will be anonymized. The generative model approach is thus pitched as mitigating privacy concerns, and is promoted for applications in fields like medicine (Goncalves et al., 2020).

The Austrian company Mostly.AI, which uses generative models to produce synthetic data for banking, insurance and telecommunications companies, describes synthetic data as data which is 'nearly indistinguishable' from the original data it was generated from but 'bears no direct relationship' to it (Pasieka, 2020). They even proclaim that 'synthetic datasets contain all the value of the data without any privacy risk. A bit like a rich cake without calories' (Mostly.AI, nd). While such claims for anonymization are disputed (Stadler, Oprisanu and Troncoso, 2021), they have convinced the USA's medical administration. In 2020, the National Institutes of Health entered into a partnership with Israeli company MDClone to produce an anonymized synthetic clinical dataset for COVID-19 research (MDClone, 2020). 


\section{Simulated Environments}

At the furthest end of the synthetic data continuum we have the use of simulated environments. In this approach, data is synthetic not because it is extrapolated from existing conventional data, but because it is collected within a synthetic world. Simulated environments can be as simple as an empty virtual space in which objects can be captured in images. The dataset ShapeNet, which features around 51,300 categorized and labeled 3D models, ranging from airplanes to wine bottles, can be inserted into such an environment. More complex simulations range from detailed indoor scenes, like Facebook's Habitat, to full cityscapes, complete with moving vehicles, pedestrians and dynamic weather, such as VIVID (Virtual Environment for Visual Deep Learning) (Lai, nd). Environments may be passive, in that one merely gathers image or video data from within them. They may also be interactive, in which some sort of agent or user can interact with them to generate data, such as Waymo's Simulation City, which generates driving data for autonomous vehicles (Hawkins 2021). The increasingly sophisticated graphics of video games have also made their simulated environments, including that of Grand Theft Auto V, attractive venues for synthetic data generation (Richter, Vineet, Roth and Koltun, 2016). Video game engines are also being used to build bespoke simulated environments. The developers of the Unity engine sell a simulated environment for autonomous vehicle training which they claim is used by ' $80 \%$ of the world's leading automotive manufacturers' (Unity Technologies, nd).

Simulated environments are the holy grail of synthetic data. Once constructed, they present the possibility of data which is not extrapolated from an existing dataset. This is desirable not only because it nullifies privacy concerns, but also the labour of data collection and labeling:

if you have a three-dimensional virtual scene complete with objects of interest, and images for the dataset are produced by rendering, it means that you automatically know which object every pixel belongs to, what are the 3D relations between them, and so on, and so forth. Producing new data becomes very cheap, and labeling becomes free (Nikolenko, 2021: v).

Yet, even as they present such advantages, simulations remain quite labour-intensive since they must be built in the first place. Even the adaptation of existing video game environments generally requires extensive labeling. Thus, the data shortage reappears within the virtual realm. Some synthetic data practitioners already speak of a lack of 'asset diversity' or paucity of virtual things (AI.Reverie, 2021). And as the ambitions of synthetic data producers grow, so will the size and complexity of simulated environments. Thus, research on the automated production of whole environments is underway using procedural generation (Qi et al., 2018) and generative adversarial models (Santana and Hotz, 2016). Microsoft researchers have recently shown that procedural generation, in combination with a large library of hand crafted 3D components, can be used to generate synthetic faces which can then be used to train facial recognition algorithms, without any real world data as input. The researchers conclude that with 'a sufficiently good 
synthetic framework, it is possible to create training data that can be used to solve real world problems in the wild, without using any real data at all' (Wood et al., 2021: 3682). This experiment demonstrates starkly the novel situation posed by synthetic data. If conventional data can be dispensed with, then so can its invasive collection. Additional layers of technological mediation and new forms of labour can replace surveillance.

\section{Data Adequate to Capital}

The approaches discussed above face a shared technical problem of whether models trained on synthetic data will work when applied to real world data. This is called the problem of syntheticto-real 'domain transfer' (Nikolenko, 2021: vi) or of bridging the 'reality gap' (Tremblay et al., 2018). All machine learning projects are concerned with 'generalization ability' or how well a model functions when applied to data it was not trained on (Alpaydin, 2016: 40). With synthetic data this concern is amplified because it is even less certain whether training data adequately emulates the real world, especially in the case of simulated environments in which there are 'limitations on rendering quality, including unrealistic texture, appearance, illumination and scene layout, etc' (Chen at al., 2020: 1). Synthetic data thus comes with a new technical problem of adequately representing the real world it presents an alternative to. But beyond the technical realm, what might the broader implications of a shift from surveillance to synthesis be for dataintensive capital and societies structured around it?

We have seen the technical and business motivations for using synthetic data, summed up as the data shortage. But in addition to ameliorating this particular technical issue deriving from the nature of machine learning, I argue that synthetic data also satisfies a tendency immanent to capital, towards rendering the valorization process autonomous from human subjectivity. Recall that Marx (1993) describes an immanent tendency of capital to replace humans with machines or to reshape 'the traditional, inherited means of labour into a form adequate to capital' (694). In so doing, capital increases its machinic component, or organic composition. I suggest that synthetic data be understood as a manifestation of rising organic composition because it involves increasing the machine element and minimizing the human element in the production of data. The data-intensive shift in capital is an ongoing episode in the rising organic composition of capital in which machine learning models are deployed to automate all kinds of analytic, predictive and managerial functions in the spheres of conception, production and circulation. But machine learning relies crucially on data collected via the surveillance of human subjects. While the subjects of surveillance are not labourers, they are human, and potentially fractious. By severing, or at least attenuating, the connection between data and surveillance, synthetic data contributes to the automatization of the automation technology machine learning. It signals a qualitative shift in how data is obtained, beyond merely automating an existing process. It reconfigures the conditions of possibility for data-intensive capital, and constitutes a data more adequate to capital. 


\section{Implications}

The data shortage refers to contemporary technical and social relations of data production based on surveillance, which are no longer adequate to data-driven capital. As popular discontent with surveillance mounts and new regulations following the EU's General Data Protection Regulation, such as Canada's (currently tabled) federal Bill C-11 Digital Charter Implementation Act, erect further barriers to conventional data collection, data-intensive capital will find these relations less and less tolerable. For that reason, it is worth considering the potential implications of synthetic data now. It seems reasonable to assume that synthetic data generation will occur alongside data collection via surveillance, rather than supplant it. For this reason, future research may find it fruitful to consider William Bogard's work on the interplay between surveillance and simulation in digital media. Bogard (1996) suggests that simulation technologies 'are forms of hypersurveillant control, where the prefix 'hyper' implies not simply an intensification of surveillance, but the effort to push surveillance technologies to their absolute limit' by prefiguring events, rather than merely recording them (4). Yet, he notes that 'surveillance and simulation, although really distinct assemblages, always mutually implicate and complicate each other's operations and development' (Bogard, 2006: 75). How exactly the simulated environments approach to synthetic data might figure into such a configuration remains to be explored. But for the sake of vividity, let us imagine a situation in which synthetic data fully replaces conventional data. Perhaps, surveillance for data collection is rendered impossible through sophisticated personal encryption systems, such as the gevulot technology in Hannu Rajaniemi's (2010) novel The Quantum Thief. In any case, in our imagined scenario, machine learning models are widely used but are trained on synthetic data generated through a combination of handcrafted elements and techniques such as simulated environments. What are the implications of this situation in which the reality gap replaces the data shortage as the central problem of data-intensive capital? I present a few, but leave their detailed exploration for future work.

A first implication concerns the ontological status of data. The senior vice president of AI and machine learning at simulated environment producer Unity derides real data as 'really just a snapshot of the situation' whereas synthetic data presents the possibility of 'augment[ing] that real world with special use cases, special situations, special events' (VB Staff, 2021). While conventional data is, as critical data scholars argue, a trace or recording, synthetic data is pitched as 'represent[ing] the real world in a very even way, better than the real world does' (VB Staff, 2021). It can include things that rarely or never happen. While machine learning is at base a mere 'learning from examples' (Offert, 2021: 16), the simulated environments approach means that examples can be constructed as needed. On this account, synthetic data improves upon conventional data in that it has a broader scope. To borrow from Andrejevic (2020), it is 'frameless' since it could potentially include anything, at least anything that can be simulated 
(106). Synthetic data is also, to borrow from Baudrillard (1994), 'hyperreal' since it quite literally involves the 'generation by models of a real without origin or reality' (1).

A second implication concerns the ontological status of embodiment. Phenomenological critiques of AI since Dreyfus (1972) have argued that intelligence does not consist of the abstract manipulation of formal symbols, but that it is rather the product of a body and perceptual apparatus which integrate diverse motor and sensory data. Brooks (1991) and others have pursued a project of situated/embodied AI which focuses on robotic agents developing intelligence by interacting with real environments. Some critical scholars who have seen in such efforts a welcome focus on the concrete, and a turn away from abstraction, have insisted on the socially emancipatory import of the link between intelligence and embodiment (Suchman, 2008; Pettersen, 2019). However, the sophistication of simulated environments throws the abstract/embodied dichotomy into question because it allows virtual embodied agents to interact with virtual environments. Agents consisting wholly of code can have a virtual body and perceptual apparatus, subject to simulated laws of physics, and can interact with complex environments as well as other agents, emulating sociality. Indeed, situated/embodied AI research now often proceeds in simulated environments (Heess et al. 2017). Embodiment is no longer irreducible to the virtual. It is rather a question of bridging the reality gap to translate virtual embodiment into the real world.

A third implication is epistemological, concerning machine learning models trained on synthetic data. A frequently made critique of machine learning is that it cannot produce or grasp novelty. A model can only recognize patterns that exist in its training data, which can only encompass things that have already happened. Speaking of generative models in particular, Offert (2021) notes that 'what there is to know is what is already known' (11). In other words, machine learning works via statistical induction and cannot make the leap of Peircean abduction, which invents the new (Pasquinelli, 2017). Thus, machine learning models break down when exposed to anomalous events or merely unfamiliar data. Synthetic data presents the option of including in training data events that have never occurred in the real world, opening up the possibility of models which are not limited to reiterating the past. If you are collecting data on driving for an autonomous vehicle system, you may find it difficult to recruit real drivers willing to experience a five car pile up. In a simulated environment, however, there is no barrier to running such a scenario thousands of times.

A fourth implication also concerns the epistemology of simulated environments. In our scenario, contemporary questions of how a given machine learning model works and what its training data were would remain. But an additional question could also arise: what was the simulated environment in which this data was generated like? Machine learning models consist of patterns extracted from training data. . How well do simulated pedestrians emulate actual pedestrians? However, as Cramer (2018) rightly argues, this is far from an objective form of analysis, since hermeneutics and bias are implicated 'at the point where data is captured, since almost any type of data acquisition requires subjective decision making' (35). This will not change in a simulated environment and may in fact be compounded as it cannot be assumed that 
all virtual worlds will adequately emulate relevant real world patterns. How is one to tell? What unexpected or unintended patterns might a model pick up from a simulated environment, particularly in the case of a complex environment with emergent properties generated by interacting agents? Nikolenko (2021) notes that 'thankfully' it is not a common endeavour to try to transfer AI agents trained in video games such as StarCraft to actual combat situations (215). But less extreme examples still present cause for concern.

A fifth implication is political. Marx (1990) saw the rising organic composition of capital in steam-powered machinery which he described as a 'mechanical monster whose body fills whole factories' (503). Today the mechanical monster's sprawl is perhaps most evident in the proliferation of huge data centers. But it also takes the form of deepening layers of software: networks, operating systems, applications and so on. Since capital became computational, its rising organic composition has involved a process of 'market-driven immanentization' (Land, 2012: 339-340) in which virtual markets progressively exclude 'transcendent elements' such as human subjectivity and replace them with 'economically programmed circuits' (Land, 2012: 341). Synthetic data aims to replace the transcendent human subject as the origin of data. Yet, the human subject and its surveillance have been a focal point for resistance to data-intensive capital. If the production of data withdraws from the real world and occurs deep in digital immanence, where can resistance to data-intensive capital, which was previously directed at the point of surveillance, occur?

\section{Conclusion}

Despite the heading of this section, this is a paper without many conclusions. Synthetic data is in a very early stage of commercialization and to try to flesh out its political economy in detail would be premature. I have, for the most part, posed rather than answered questions. I have aimed only to present a level-headed assessment of synthetic data, some ways in which it is produced and provide some provocative musings on its potential implications within dataintensive capitalism. While much more remains to be investigated, it is safe to conclude one thing. In response to the assumption that the source of data is necessarily human, we can offer a qualified negative. It is a qualified negative because synthetic data is far from being produced without any human intervention. Yet, it is a negative because machine learning models can indeed be trained on data which is not a recording of a human subject. Compounding layers of technological mediation-procedural generation, simulated environment, machine learning-can emulate the human as a source of data. As it turns out, data is not necessarily people, after all.

\section{Notes}

1. An interest in data is not new to capitalism. At least since the neoliberal intensification of capitalism in the 1960s, capital has had an 'intense interest in and pursuit of information technologies' for decision-making (Harvey, 2007: 3). Data-intensive capitalism amplifies the neoliberal impulse towards data collection that predates it. 
2. Much scientific data, such as that produced by particle colliders, is of limited commercial interest (for the moment).

3. One can assume that this acquisition is related to Facebook's 2021 rebranding as Meta and projected evolution into virtual reality platform Metaverse. Metaverse will require infrastructure for the automated production of simulated environments and objects since it is planned to be highly customizable even for the non-technical user.

\section{References}

AI.Reverie (2021) Train Smarter With Synthetic Data and Domain Adaptation. Available at: https://www.youtube.com/watch?v=Qs5ktD5CqJA

Alpaydin E (2016) Machine Learning: The New AI. Cambridge: MIT Press.

Andrejevic M (2013) Infoglut: How Too Much Information Is Changing the Way We Think and Know. New York: Routledge.

Andrejevic M (2020) Automated Media. New York: Routledge.

Andrews G (2021) What is synthetic data?. The Official NVIDIA Blog. Available at: https://blogs.nvidia.com/blog/2021/06/08/what-is-synthetic-data/

Bansal A, Sharma R and Kathuria M (2021) A Systematic Review on Data Scarcity Problem in Deep Learning: Solution and Applications. ACM Computing Surveys. https://dl.acm.org/doi/10.1145/3502287

Baudrillard J (1994) Simulacra and Simulation. Ann Arbor: University of Michigan Press.

Bogard W (1996) The Simulation of Surveillance: Hypercontrol in Telematic Societies. Cambridge, UK: Cambridge University Press.

Bogard W (2006) Welcome to the Society of Control: The Simulation of Surveillance Revisited. In Ericson RV and Haggerty KD (eds) The New Politics of Surveillance and Visibility. Toronto: University of Toronto Press.

Bowker GC (2005) Memory Practices in the Sciences. Cambridge, MA: MIT Press.

Braverman H (1998) Labor and Monopoly Capital: The Degradation of Work in the Twentieth Century. New York: NYU Press.

Brooks R (1991) Intelligence Without Representation. Artificial Intelligence 47(1-3): 139-159.

Caffentzis G (2012) In Letters of Blood and Fire: Work, Machines, and the Crisis of Capitalism. Oakland, CA: PM Press.

Chen W, Yu Z, Wang Z and Anandkumar A (2020) Automated Synthetic-to-Real Generalization. arXiv:2007.06965 [cs, stat] [Preprint]. Available at: http://arxiv.org/abs/2007.06965

Cheney-Lippold J (2017) We Are Data: Algorithms and the Making of Our Digital Selves. New York: NYU Press.

Chun WHK (2018) Queerying Homophily. In Pattern Discrimination. Minneapolis: University of Minnesota Press/Meson Press.

Chung J (2021) Big Tech, Big Cash: Washington's New Power Players. Public Citizen. https://www.citizen.org/article/big-tech-lobbying-update/

Cramer F (2018) Crapularity Hermeneutics: Interpretation as the Blind Spot on Analytics, Artificial Intelligence, and Other Algorithmic Producers of the Postapocalyptic Present. In Pattern Discrimination. Minneapolis: University of Minnesota Press/Meson Press.

Crawford K (2021) The Atlas of AI: Power, Politics, and the Planetary Costs of Artificial Intelligence. New Haven, CT: Yale University Press. 
DeepLearningAI (2021) A Chat with Andrew on MLOps: From Model-centric to Data-centric AI. Available at: https://www.youtube.com/watch?v=06-AZXmwHjo

Delfanti A and Frey B (2021) Humanly Extended Automation or the Future of Work Seen through Amazon Patents. Science, Technology, \& Human Values 46(3): 655-682.

Dilemgani C (2020) Synthetic Data Generation: Techniques, Best Practices \& Tools. Available at: https://research.aimultiple.com/synthetic-data-generation/

Dimensional Research (2019) Artificial Intelligence and Machine Learning Projects Are Obstructed by Data Issues. Available at: https://cdn2.hubspot.net/hubfs/3971219/Survey\%20Assets\%201905/Dimensional\%20Res earch\%20Machine\%20Learning\%20PPT\%20Report\%20FINAL.pdf

Dippel A (2021) Metaphors We Live By. In Sudmann A (ed) The Democratization of Artificial Intelligence: Net Politics in the Era of Learning Algorithms. Bielefeld: transcript.

Draper NA and Turow J (2019) The Corporate Cultivation of Digital Resignation. New Media \& Society 21(8): 1824-1839.

Dreyfus H (1972) What Computers Can't Do: The Limits of Artificial Intelligence. New York: Harper \& Row.

Dyer-Witheford N, Kjøsen AM and Steinhoff J (2019) Inhuman Power: Artificial Intelligence and the Future of Capitalism. London: Pluto Press.

El Emam K (2021) Could Synthetic Data Be the Future of Data Sharing?. CPO Magazine. Available at: https://www.cpomagazine.com/data-privacy/could-synthetic-data-be-thefuture-of-data-sharing/

Foraker RE, Yu SC, Gupta A, et al. (2020) Spot the difference: comparing results of analyses from real patient data and synthetic derivatives. JAMIA Open 3(4): 557-566.

Gerard A (2021) What is synthetic data?. The Official NVIDIA Blog. Available at: https://blogs.nvidia.com/blog/2021/06/08/what-is-synthetic-data/

Gitelman L (ed.) (2013) 'Raw Data' Is an Oxymoron. Cambridge, MA: MIT Press.

Goncalves A, Ray P, Soper B, et al. (2020) Generation and evaluation of synthetic patient data. BMC Medical Research Methodology 20(1).

Goodfellow I, Pouget-Abadie J, Mirza M, et al. (2014) Generative Adversarial Nets. In Ghahramani Z (ed.) Advances in Neural Information Processing Systems 27. Red Hook, NY: Curran Associates Inc. Available at: https://proceedings.neurips.cc/paper/2014/hash/5ca3e9b122f61f8f06494c97b1afccf3Abstract.html

Gray ML and Suri S (2019) Ghost Work: How to Stop Silicon Valley from Building a New Global Underclass. San Francisco, CA: HMH Books.

Gregory K (2014) Big data, like Soylent Green, is made of people. Digital Labor Working Group. Available at: https://digitallabor.commons.gc.cuny.edu/2014/11/05/big-data-likesoylent-green-is-made-of-people/

Halpern O (2021) Planetary Intelligence. In Roberge J and Castelle M (eds) The Cultural Life of Machine Learning. Cham: Palgrave Macmillan.

Harvey D (2007) A Brief History of Neoliberalism. Oxford: Oxford University Press.

Hawkins AJ (2021) Welcome to Simulation City, the virtual world where Waymo tests its autonomous vehicles. The Verge. Available at: https://www.theverge.com/2021/7/6/22565448/waymo-simulation-city-autonomousvehicle-testing-virtual 
Heess N, TB D, Sriram S, et al. (2017) Emergence of Locomotion Behaviours in Rich Environments. arXiv preprint:1707.02286

Incze R (2019) The Cost of Machine Learning Projects. Cognifeed. Available at: https://medium.com/cognifeed/the-cost-of-machine-learning-projects-7ca3aea03a5c

Katz Y (2020) Artificial Whiteness: Politics and Ideology in Artificial Intelligence. New York: Columbia University Press.

Kitchin R (2014) Big Data, new epistemologies and paradigm shifts. Big Data \& Society 1(1).

Krizhevsky A, Sutskever I, and Hinton GE (2012) ImageNet Classification with Deep Convolutional Neural Networks. In Pereira F et al. (ed.) Advances in Neural Information Processing Systems 25. Red Hook, NY: Curran Associates Inc. Available at: https://proceedings.neurips.cc/paper/2012/hash/c399862d3b9d6b76c8436e924a68c45bAbstract.html

Land N (2012) Fanged Noumena: Collected Writings 1987-2007. Falmouth: Urbanomic.

Lai KT (2021) VIVID - Virtual Environment for Visual Deep Learning. Available at: https://github.com/kuanting/vivid

Lemley J, Bazrafkan S, and Corcoran P (2017) Smart Augmentation: Learning an Optimal Data Augmentation Strategy. IEEE Access 5: 5858-5869.

Lemov R (2016) Why big data is actually small, personal and very human. Aeon. Available at: https://aeon.co/essays/why-big-data-is-actually-small-personal-and-very-human

Lepage-Richer T (2021) Adversariality in Machine Learning Systems: On Neural Networks and the Limits of Knowledge. In Roberge J and Castelle M (eds) The Cultural Life of Machine Learning. Cham: Palgrave Macmillan.

Lucini F (2021) The Real Deal About Synthetic Data. MIT Sloan Management Review. Available at: https://sloanreview.mit.edu/article/the-real-deal-about-synthetic-data/

Manovich L (2001) The Language of New Media. Cambridge, MA: MIT Press.

Marx K (1990) Capital Volume I. New York: Penguin

Marx K (1993) Grundrisse: Foundations of the Critique of Political Economy. New York: Penguin.

MDClone (nd) MDClone and National Institutes of Health (NIH) to Support National Scientific Exploration for COVID-19 Efforts. Available at: https://www.mdclone.com/newspress/articles/mdclone-and-national-institutes-of-health

Microsoft (nd) AI Lab Project: Synthetic Data Generator. https://www.microsoft.com/enus/ai/ai-lab-synthetic-data-showcase

Mirrlees T and Alvi S (2019) EdTech Inc.: Selling, Automating and Globalizing Higher Education in the Digital Age. New York: Routledge.

Mosco V (2015) To the Cloud: Big Data in a Turbulent World. New York: Routledge.

Mostly.AI (nd) The definitive guide to synthetic data. Available at: https://mostly.ai/syntheticdata/

Nikolenko SI (2021) Synthetic Data for Deep Learning. Cham: Springer Nature.

Noble DF (1986) Forces of Production: A Social History of Industrial Automation. Oxford: Oxford University Press.

Noble SU (2018) Algorithms of Oppression: How Search Engines Reinforce Racism. New York: NYU Press.

Ntoutsi E, Fafalios P, Gadiraju U, et al. (2020) Bias in data-driven artificial intelligence systems-An introductory survey. WIREs Data Mining and Knowledge Discovery 10(3). 
Offert F (2021) Latent Deep Space: Generative Adversarial Networks (GANs) in the Sciences. Media+Environment 3(2).

Pasieka M (2020) The evolution of synthetic data: a comparison of three data generation methods. Available at: https://mostly.ai/blog/comparison-of-synthetic-data-types/

Pasquinelli M (2017) Machines that Morph Logic: Neural Networks and the Distorted Automation of Intelligence as Statistical Inference. Glass Bead 1.

Patki N, Wedge R and Veeramachaneni K (2016) The Synthetic Data Vault. In 2016 IEEE International Conference on Data Science and Advanced Analytics (DSAA): 399-410. Red Hook, NY: Curran Associates Inc.

Pettersen L (2019) Why Artificial Intelligence Will Not Outsmart Complex Knowledge Work. Work, Employment and Society 33(6): 1058-1067.

Qi S, Zhu Y, Huang S, et al. (2018) Human-centric indoor scene synthesis using stochastic grammar. In: 2018 IEEE/CVF Conference on Computer Vision and Pattern Recognition: 5899-5908. Red Hook, NY: Curran Associates Inc.

Raghunathan TE (2021) Synthetic Data. Annual Review of Statistics and Its Applications 8: 129140.

Rajaniemi H (2010) The Quantum Thief. London: Victor Gollancz Ltd.

Ramtin R (1991) Capitalism and Automation: Revolution in Technology and Capitalist Breakdown. London: Pluto Press.

Rankin D, Black M, Bond R, et al. (2020) Reliability of Supervised Machine Learning Using Synthetic Data in Health Care: Model to Preserve Privacy for Data Sharing. JMIR Medical Informatics 8(7): e18910.

Roberge J and Castelle M (2021) Toward an End-to-End Sociology of 21st-Century Machine Learning. In Roberge J and Castelle M (eds) The Cultural Life of Machine Learning. Cham: Palgrave Macmillan.

Richter SR, Vineet V, Roth S and Koltun V (2016) Playing for Data: Ground Truth from Computer Games. In Leibe B et al. (eds) Computer Vision - ECCV 2016. Cham: Springer International Publishing.

Ross J (2015) Piketty and Marx's Rising Organic Composition of Capital: Review of Capital in the Twenty-First Century by Thomas Piketty. International Critical Thought 5(2):241257.

Sadowski J (2019) When data is capital: Datafication, accumulation, and extraction. Big Data \& Society 6(1).

Santana E and Hotz G (2016) Learning a Driving Simulator. arXiv:1608.01230 [cs, stat] [Preprint]. Available at: http://arxiv.org/abs/1608.01230

Srnicek N (2017) Platform Capitalism. London: Polity.

Stadler T, Oprisanu B and Troncoso C (2021) Synthetic Data -- Anonymisation Groundhog Day. arXiv:2011.07018 [cs] [Preprint]. Available at: http://arxiv.org/abs/2011.07018

Steinhoff J (2021) Automation and Autonomy: Labour, Capital and Machines in the Artificial Intelligence Industry. London: Palgrave Macmillan.

Suchman L (2007) Feminist STS and the Sciences of the Artificial. In Hacket E, et al. (eds.) The Handbook of Science and Technology Studies, Third Edition. Cambridge, MA: MIT Press.

Sudmann A (ed) (2019) The Democratization of Artificial Intelligence: Net Politics in the Era of Learning Algorithms. Bielefeld: transcript. 
Unity Technologies (nd) Autonomous Vehicle Training with Simulated Environments. Available at: https://unity.com/how-to/simulated-environments-for-autonomous-vehicle-training

Tremblay J, Prakash A, Acuna D, et al. (2018) Training Deep Networks with Synthetic Data: Bridging the Reality Gap by Domain Randomization. In 2018 IEEE/CVF Conference on Computer Vision and Pattern Recognition Workshops (CVPRW). Red Hook, NY: Curran Associates Inc.

VB Staff (2021) Unity's Danny Lange explains why synthetic data is better than the real thing at Transform 2021. VentureBeat. 12 July. Available at: https://venturebeat.com/2021/07/12/unitys-danny-lange-explains-why-synthetic-data-isbetter-than-the-real-thing-at-transform-2021-2/

Verdegem P (ed) (2021) AI for Everyone? Critical Perspectives. London: University of Westminster Press.

Watson A (2020) Reducing AI bias with Synthetic data. Available at: https://towardsdatascience.com/reducing-ai-bias-with-synthetic-data-7bddc39f290d

West SM (2019) Data Capitalism: Redefining the Logics of Surveillance and Privacy. Business \& Society 58(1): 20-41.

Wiggers K (2021a) Facebook quietly acquires synthetic data startup AI.Reverie. VentureBeat. 12 October. Available at: https://venturebeat.com/2021/10/11/facebook-quietly-acquiressynthetic-data-startup-ai-reverie/

Wiggers K (2021b) MIT study finds 'systematic' labeling errors in popular AI benchmark datasets. VentureBeat. 29 March. Available at: https://venturebeat.com/2021/03/28/mitstudy-finds-systematic-labeling-errors-in-popular-ai-benchmark-datasets/

Wood E, Baltrušaitis T, Hewitt C, et al. (2021) Fake It Till You Make It: Face Analysis in the Wild Using Synthetic Data Alone. International Conference on Computer Vision 2021. Available at: https://arxiv.org/abs/2109.15102v2

Yang Q (2019) GDPR, Data Shortage and AI. Available at: https://www.swissre.com/dam/jcr:2edc3963-3226-4ce4-aee68496c0247b63/Smart_resilience_Qiang\%20Yang_ENGLISH.pdf

Zuboff S (2016). The secrets of surveillance capitalism. Frankfurter Allgemeine. Available at: http://www.faz.net/aktuell/feuilleton/debatten/the-digital-debate/shoshana-zuboff-secretsof-surveillance-capitalism14103616.html

Zuboff S (2019) The Age of Surveillance Capitalism: The fight for a human future at the new frontier of power. London: Profile Books. 